\title{
ON THE EXPONENTIAL BEHAVIOR OF STOCHASTIC EVOLUTION EQUATIONS FOR NON-NEWTONIAN FLUIDS
}

\author{
PAUL ANDRÉ RAZAFIMANDIMBY AND MAMADOU SANGO
}

\begin{abstract}
We investigate the exponential long-time behaviour of the stochastic evolution equations describing the motion of a Non-Newtonian fluids excited by multiplicative noise. Some results on the exponential convergence in mean square and with probability one of the weak probabilistic solution to the stationary solutions are given. We also prove an interesting result related to the stabilization of these stochastic evolution equations.
\end{abstract}

\section{INTRODUCTION}

For a homogeneous incompressible fluid, the constitutive law satisfies

$$
\mathbb{T}=-\tilde{p} \mathbf{1}+\hat{\mathbb{T}}(\mathbf{E}(u))
$$

where $\mathbb{T}$ is the Cauchy stress tensor, $u$ is the velocity of the fluid, and $\pi$ is the undetermined pressure due to the incompressibility condition, $\mathbf{1}$ is the identity tensor. The argument tensor $\mathcal{E}(u)$ of the symmetric-valued function $\hat{\mathbb{T}}$ is defined through

$$
\mathcal{E}(u)=\frac{1}{2}\left(\mathbf{L}+\mathbf{L}^{\mathrm{T}}\right), \quad \mathbf{L}=\operatorname{grad} u,
$$

where the $\mathrm{T}$ superscript denotes the matrix transpose. The Navier-Stokes equations (NSE) describe the equations of motion of Newtonian model of fluids which imposes that $\hat{\mathbb{T}}$ is a linear function of $\mathcal{E}(u)$. Many scientists believe that NSE is an accurate model for the motion of incompressible viscous fluids in many practical situations, and even for turbulent flows. However, there are a lot of materials such as polymer solutions, visco-elastic or visco-plastic fluids, paints and industrial fluids, etc, that cannot be characterized by the NSE. To describe these materials one generally has to use fluids model that allows $\hat{\mathbb{T}}$ being a nonlinear function of $\mathcal{E}(u)$. Fluids in the latter class are called Non-Newtonian fluids. In [24] and [25], Ladyzhenskaya considered a model of nonlinear fluids whose reduced stress tensor $\hat{\mathbb{T}}(\mathcal{E}(u))$ satisfies

$$
\hat{\mathbb{T}}(\mathcal{E}(u))=2\left(\varepsilon+\mu_{0}|\nabla u|^{r}\right) \frac{\partial u_{i}}{\partial x_{j}}, r>0 .
$$

Since then, this model has been the object of intense mathematical analysis which have generated several important results. We refer to [20], [26] for some relevant examples. In [20] the authors emphasized important reasons for considering such model. Recently Necas, Novotny and Silhavy [31], Bellout, Bloom and Necas [1] has developed the theory of multipolar viscous fluids which was based on the work of Necas and Silhavy [30]. Their theory is compatible with the basic principles of thermodynamics such as the Clausius-Duhem inequality and the principle of frame indifference, and their results up to date indicate that the theory of multipolar fluids may lead to a better understanding of hydrodynamic turbulence (see for example [4]). In this paper, we are interested in the stochastic version of the equations of motion of a particular class of multipolar fluids whose reduced stress tensor $\hat{\mathbb{T}}(\mathcal{E}(u))=\tau(\mathcal{E}(u))$ is given by

$$
\tau(\mathcal{E}(u))=2 \mu_{0}\left(\varepsilon+|\mathcal{E}(u)|^{2}\right)^{\frac{p-2}{2}} \mathcal{E}(u)-2 \mu_{1} \Delta \mathcal{E}(u) .
$$

2000 Mathematics Subject Classification. 60H15, 35Q35, 60H30, 35R15.

Key words and phrases. Stochastic evolution equations, Weak solution, Asymptotic behaviour, Non-Newtonian fluids, Bipolar fluids, Stabilization. 
More precisely, let $n=2,3, D \subset \mathbb{R}^{n}$ be a smooth bounded open domain, we consider

$$
\left\{\begin{array}{l}
d u+[u \cdot \nabla u-\nabla \cdot \tau(\mathcal{E}(u))+\nabla \pi] d t=F(u) d t+G(t, u) d W, x \in D, t \in(0, t] \\
u(x, 0)=u_{0}, x \in D \\
\nabla \cdot u=0, x \in D, t \in[0, T] \\
u(x, t)=\tau_{i j l} n_{j} n_{l}=0, x \in \partial D, t \in(0, T)
\end{array}\right.
$$

where $u$ is the velocity of the fluids, $\pi$ its pressure, $n$ denotes the normal exterior to the boundary and

$$
\begin{aligned}
& \mathcal{E}(u)=\frac{1}{2}\left(\frac{\partial u_{i}}{\partial x_{j}}+\frac{\partial u_{j}}{\partial x_{i}}\right),|\mathcal{E}(u)|^{2}=\sum_{i, j=1}^{n}\left|\mathcal{E}_{i j}(u)\right|^{2}, \\
& \tau(\mathcal{E}(u))=2 \mu_{0}\left(\varepsilon+|\mathcal{E}(u)|^{2}\right)^{\frac{p-2}{2}} \mathcal{E}(u)-2 \mu_{1} \Delta \mathcal{E}(u) .
\end{aligned}
$$

The quantities $\varepsilon, \mu_{0}$ and $\mu_{1}$ denote positive constants. Here $W$ is a cylindrical Wiener process for white noise defined on probability space $(\Omega, \mathcal{F}, \mathbb{P})$ and taking its values in a separable Hilbert space $H$ to be defined later. The system (1) describes the equations of motion of isothermal incompressible nonlinear bipolar fluids excited by random forces.

For $p=2, \mu_{1}=0, G \equiv 0,(1)$ is the Navier-Stokes equations which has been extensively studied (see, for instance, [40]). If $1<p<2$ then the fluid is shear thinning, and it is shear thickening when $2<p$. The problem (1) is as interesting as the Navier-Stokes equations. It contains two nonlinear terms which makes the problem as difficult as any nonlinear evolution equations. During the last two decades, the deterministic version of (1) has been the object of intense mathematical investigation which has generated several important results. We refer to [2], [3], [5], [27], [28] for relevant examples. Despite these numerous results there are still a lot of open problems related to the mathematical theory of multipolar fluids. Some examples are the existence of weak solution for all values of $p$, the uniqueness of such weak solutions and many more. We refer, for instance, to [3], [21] and [28] for some discussions about these challenges.

Since the pioneering work of Bensoussan and Temam [8] on stochastic Navier-Stokes equations, stochastic partial differential equations and stochastic models for Newtonian fluid dynamics have been the object of intense investigations which have generated several important results. We refer, for instance, to [6], [7],[9], [10], [11], [12], [13], [14], [16],[17], [18], [19], [29], [32], [33],[37], $[38],[39]$. However, there are only very few results for the dynamical behaviour of stochastic models for Non-Newtonian fluids (see [15], [23], [34], [35], [36]). In [23] the authors proved the existence of martingale and stationary solution of (1) for the case $1<p$. Existence and uniqueness of strong probabilistic solution to (1) is proved in [15] for the case of nonlinear bipolar fluids, i.e, for $1<p<2$.

The analysis of the exponential behaviour of the weak solution of a fluid model as $t \rightarrow \infty$ is amongst the most interesting and important problems in mathematical theory of fluids dynamics (see for example [12] and [14]). But it seems that there is no known result on this topic for stochastic model of Non-Newtonian fluids. In this paper, we are interested in filling that gap, and to do so we consider (1) with $G \neq 0$ and $2<p$. More precisely, we are aiming to investigate on the exponential convergence in mean square and almost surely of the weak probabilistic solutions to the stationary solution. By following the method in [12] and [14], we mainly prove that under various conditions on the forcing terms $F$ and $G$, the exponential convergence in mean square and almost surely of the weak probabilistic solution to the stationary solution holds. We also show that there is a stabilization effect of the stochastic perturbation under some conditions on the noise term and the physical parameters such as $\mu_{0}$ and $\mu_{1}$ for instance. It should be noted that the problem we treated here does not fall into the general framework considered in [12] or in [14].

The rest of the paper is structured as follows. The next section is devoted to the mathematical setting of the problem. We prove in section 3 some results on the exponential convergence in 
mean square and almost surely of the weak probabilistic solution. In the last section we discuss on the stabilization effect of the stochastic excitation.

\section{Mathematical Settings of the problem (1)}

Throughout this paper we mainly use the same notations as in [23]. By $L^{q}(D)$ we denote the Lebesgue space of $q$-th integrable functions with norm $\|\cdot\|_{L^{q}}$. For the particular case $q=2$, we denote its norm by $\|\cdot\|$. For $q=\infty$ the norm is defined by $\|\cdot\|_{L^{\infty}}=\operatorname{ess}_{\sup } \operatorname{su}_{x \in D}|u(x)|$, where $|x|$ is the euclidean norm of the vector $x \in \mathbb{R}^{n}$. The Sobolev space $\left\{u \in L^{q}(D): D^{k} u \in L^{q}(D), k \leq \sigma\right\}$ with norm $\|\cdot\|_{q, \sigma}$ is denoted by $W^{q, \sigma}$. $C(I, X)$ is the space of continuous functions from the interval $I=[0, T]$ to $X$, and $L^{q}(I, X)$ is the space of all measurable functions $u:[0, T] \rightarrow X$, with the norm

$$
\|u\|_{L^{q}(I, X)}^{q}=\int_{0}^{T}\|u(t)\|_{X}^{q} d t, q \in[1, \infty),
$$

and when $q=\infty,\|u\|_{L^{\infty}(I, X)}=\operatorname{ess}_{\sup _{t \in[0, T]}}\|u(t)\|_{X}$.

In a very similar manner, we also define the space $L^{q}(\Omega, X)$. The mathematical expectation associated to the probability space $(\Omega, \mathcal{F}, \mathbb{P})$ is denoted by $\mathbb{E}$.

We proceed with the definitions of some additional spaces frequently used in this work. We define a space of functions with support strictly contained in $D$ and satisfying the divergence free condition:

$$
\mathcal{V}=\left\{u \in \mathcal{C}_{c}^{\infty}(D): \nabla \cdot u=0\right\} .
$$

We denote by $H$ the closure of $\mathcal{V}$ with norm $\|\cdot\|$ in $L^{2}(D)$. It is a Hilbert space when equipped with the $L^{2}(D)$-inner product $(.,$.$) . H^{\sigma}(D)$ is the closure of $\mathcal{V}$ in $W^{2, \sigma}$ with the norm $\|\cdot\|_{\sigma}$, and $H^{-\sigma}$ is the dual space $(\sigma \geq 1)$. If $\sigma=2$, then $V=H^{2}(D)$ and $V^{*}$ is the dual space of $V$. The duality product between $V$ and $V^{*}$ is denoted by $\left.<.,.\right\rangle$. We assume throughout that there exists a positive constant $\lambda_{1}$ such that that the Poincaré inequalities type

$$
\|u\|_{\sigma}^{2} \leq \frac{1}{\lambda_{1}}\|u\|_{\sigma+1}^{2},
$$

hold for any $u \in H^{\sigma+1}, \sigma \geq 0$.

Note that $H$ is a separable Hilbert space, so we can associate with it an orthonormal basis $\left\{e_{i}: i=1,2, \ldots\right\}$. The function $\left\{W_{t}: t \in[0, T]\right\}$ defines a cylindrical Wiener process

$$
W_{t}=\sum_{i=1}^{\infty} \mathcal{W}_{t}^{i}
$$

where $\left(\mathcal{W}_{t}^{i}\right)_{i=1,2, \ldots}$ is a sequence of mutually independent one dimensional Wiener processes. The space of all Hilbert-Schmidt operators from $H$ to $H^{\sigma}(D)$ is denoted by $L_{2}^{0, \sigma}, \sigma \geq 0$. Throughout this paper, we assume that $G$ is a $L_{2}^{0,0}$-valued continuous mapping defined on $[0, T] \times H$.

We will rewrite (1) in the following equivalent form

$$
\left\{\begin{array}{l}
d u+\left[\mu_{1} A u+2 \mu_{0} A_{p} u+B(u, u)\right] d t=F(u) d t+G(t, u) d W \\
u(0)=u_{0} .
\end{array}\right.
$$

Here the operator $A$ is defined through the relation

Note that

$$
<A u, v>=a(u, v)=\int_{D} \frac{\partial \mathcal{E}_{i j}(u)}{\partial x_{k}} \frac{\partial \mathcal{E}_{i j}(v)}{\partial x_{k}} d x, u \in D(A), v \in V .
$$

$$
D(A)=\left\{u \in V: a(u, v)=(f, v), f \in H \subset V^{*}, \forall v \in V\right\},
$$

$A=\mathbf{P} \Delta^{2}$, where $\mathbf{P}$ is the orthogonal projection defined on $L^{2}(D)$ onto $H$.

We also introduce a bilinear form $B(u, v): H^{1}(D) \times H^{1}(D) \rightarrow H^{-1}$ as follows:

$$
(B(u, v), w)=b(u, v, w)=\int_{D} u_{i} \frac{\partial v_{j}}{\partial x_{i}} w_{j} d x, u, v, w \in H^{1}(D),
$$


where $b(., .,$.$) is the well-known trilinear form used in the mathematical analysis of Navier-Stokes$ equations (see for instance [40]). The bilinear form $B(\cdot, \cdot)$ enjoys the following properties:

- for any $u, v, w \in H^{1}(D)$, we have

$$
(B(u, v), w)=-(B(u, w), v) \text { and }(B(u, v), v)=0 .
$$

- There exists a constant $C_{0}$ such that

$$
|(B(u, v), u)| \leq C_{0}\|u\|_{2}^{2}\|v\|_{1}
$$

for any $u, v \in V$.

The inequality (5) can be proved by using the Hölder's and Sobolev inequalities (see [40]). The nonlinear term $A_{p}: V \rightarrow V^{*}$ is defined as follows:

$$
\left(A_{p} u, v\right)=\int_{D} \Gamma(u) \mathcal{E}_{i j}(u) \mathcal{E}_{i j}(v) d x, u, v \in V
$$

where $\Gamma(u)=\left(\varepsilon+|\mathcal{E}(u)|^{2}\right)^{\frac{p-2}{2}}$. Some of the properties of $A_{p}$ is given below.

Lemma 2.1. $\quad$ (i) $A_{p}$ is locally Lipschitz continuous, i.e., there exists $C>0$ such that

$$
\left\|A_{p} u-A_{p} v\right\|_{V^{*}} \leq C\|u-v\|_{2}, u, v \in V .
$$

(ii) If $u \in L^{2}(0, T, V) \cap L^{\infty}(0, T, H)$, then $A_{p} u \in L^{2}\left(0, T, V^{*}\right)$.

(iii) There exists a positive constant $K_{0}$ such that

$$
\left(A_{p} u-A_{p} v, u-v\right) \geq \varepsilon^{\frac{p-2}{2}}\|u-v\|_{1},
$$

for any $u, v \in V$.

The point (i) and (ii) were proved in [23], and (iii) in [20] for example. From now on, we will work with (3). (3).

We define very clearly what we mean by weak probabilistic solution (weak solution) of (1) or

Definition 2.2. A weak solution to $(3)$ is a system $\left(\Omega, \mathcal{F}, \mathbb{P},\left(\mathbb{F}^{t}\right)_{t \in[0, T]}, W, u\right)$ such that

(1) $\left(\Omega, \mathcal{F}, \mathbb{P},\left(\mathbb{F}^{t}\right)_{t \in[0, T]}, W\right)$ is a stochastic basis,

(2) $u$ is $\mathbb{F}^{t}$-adapted,

(3) $u \in C\left(0, T, H^{-3}\right) \cap L^{2}(0, T, V) \cap L^{\infty}(0, T, H)$ with probability one,

(4) for all $t \in[0, T]$ and $v \in H^{3}(D)$ the following holds almost surely

$$
\begin{aligned}
(u(t), v)= & \left.\left(u_{0}, v\right)-\mu_{1} \int_{0}^{t}(A u(s), v)\right) d s-\int_{0}^{t}(B(u(s), u(s)), v) d s \\
& -2 \mu_{0} \int_{0}^{t}\left(A_{p} u(s), v\right) d s+\int_{0}^{t}(F(u), v) d s+\left(\int_{0}^{t} G(s, u(s)) d W, v\right) .
\end{aligned}
$$

In the present work, we are interested in the asymptotic behavior of weak probabilistic solutions of (3) so we will assume the existence of such solution. We refer to [15] and [23] for the existence and uniqueness of the weak and strong probabilistic solution to (3).

Next we give the following definitions which are taken from [12] and [14].

Definition 2.3. We say that a weak solution $u(t)$ to (3) converges $u_{\infty}$ exponentially in mean square if there exist $a>0$ and $M_{0}=M_{0}\left(u_{0}\right)>0$ (which may depend on $u_{0}$ ) such that

$$
\mathbb{E}|| u(t)-u_{\infty} \|^{2} \leq M_{0} e^{-a t}, t \geq 0 .
$$

In particular, if $u_{\infty}$ is a solution to (3) then it is said that $u_{\infty}$ is exponentially stable in the mean square provided that any weak solution $u(t)$ to (3) converges to $u_{\infty}$ exponentially in mean square with the same exponential order $a>0$. 
Definition 2.4. We say that a weak solution $u(t)$ to $(3)$ converges to $u_{\infty}$ almost surely exponentially if there exists $\eta>0$ such that

$$
\lim _{t \rightarrow \infty} \frac{1}{t} \log \left\|u(t)-u_{\infty}\right\|^{2} \leq-\eta \text { almost surely. }
$$

In particular, if $u_{\infty}$ is a solution to (3) then it is said that $u_{\infty}$ is almost surely exponentially stable provided that any weak solution $u(t)$ to $(3)$ converges to $u_{\infty}$ exponentially with probability one with the same constant $\eta>0$.

\section{Exponential Stability of WEAK PROBABilistic SOLUtions}

In this section we discuss the mean square and almost sure exponential stability of the stationary solution $u_{\infty}$ associated to (3). For the major part of this section we will assume that:

Conditions $\mathrm{F} 1$. there exists a positive constant $\beta$ such that

$$
\begin{gathered}
F(0) \neq 0, \\
\|F(u)-F(v)\|_{V^{*}} \leq \beta\|u-v\|_{2}, u, v \in V .
\end{gathered}
$$

Now, let us consider the stationary equations

$$
\left\{\begin{array}{l}
u_{\infty} \cdot \nabla u_{\infty}-\nabla \cdot \tau\left(\mathcal{E}\left(u_{\infty}\right)\right)+\nabla \pi=F\left(u_{\infty}\right), x \in D, \\
\nabla \cdot u=0, x \in D, \\
u(x)=\tau_{i j l} n_{j} n_{l}=0, x \in \partial D .
\end{array}\right.
$$

With the notations introduced in the previous section, (8) can be rewritten in the following form

$$
\mu_{1} A u_{\infty}+2 \mu_{0} A_{p} u_{\infty}+B\left(u_{\infty}, u_{\infty}\right)=F\left(u_{\infty}\right) .
$$

The next result is about the existence and uniqueness of the solution $u_{\infty}$ of (9).

Theorem 3.1. Assume that Conditions F1 hold.

(i) If $\mu_{1}-\beta>0$ then there exists a solution $u_{\infty}$ to (9) such that

$$
\left\|u_{\infty}\right\|_{1} \leq \frac{\|F(0)\|_{V^{*}}}{\mu_{1}-\beta}
$$

(ii) If in addition

$$
\mu_{1}>\frac{C_{0}\|F(0)\|_{V^{*}}}{\sqrt{\lambda_{1}}\left(\mu_{1}-\beta\right)}+\beta
$$

then the stationary solution $u_{\infty}$ of $(9)$ is unique.

Proof. This result was proven in [22] by the use of the Brouwer fixed point theorem for the case $F(u)=f(x) \in H$. Here, we will just prove (10) and show how (11) is related to the uniqueness of $u_{\infty}$.

If $u_{\infty}$ is a solution of (9) then we can check that it satisfies

$$
\mu_{1}\left\|u_{\infty}\right\|_{2}^{2}+2 \mu_{0}\left(A_{p} u_{\infty}, u_{\infty}\right)+\left(B\left(u_{\infty}, u_{\infty}\right), u_{\infty}\right)=<F\left(u_{\infty}\right), u_{\infty}>.
$$

By using (4), (7) and Conditions F1 we infer from the above equation that

$$
\mu_{1}|| u_{\infty}\left\|_{2}^{2} \leq\left(\beta\left\|u_{\infty}\right\|_{2}+\|F(0)\|_{V^{*}}\right)\right\| u_{\infty} \|_{2},
$$

from which we deduce that

$$
\left\|u_{\infty}\right\|_{2} \leq \frac{\|F(0)\|_{V^{*}}}{\mu_{1}-\beta}
$$

It makes sense since $\mu_{1}-\beta>0$ by assumption.

Now, let $u_{\infty}^{1}$ and $u_{\infty}^{2}$ be two solutions of (9) and let us set $w=u_{\infty}^{1}-u_{\infty}^{2}$. We can check that the following holds

$$
\mu_{1} A w+2 \mu_{0}\left(A-p u_{\infty}^{1}-A_{p} u_{\infty}^{2}\right)+B\left(u_{\infty}^{1}, u_{\infty}^{1}\right)-B\left(u_{\infty}^{2}, u_{\infty}^{2}\right)=F\left(u_{\infty}^{1}\right)-F\left(u_{\infty}^{2}\right) .
$$


Multiplying this equation by $w$ yields

$$
\mu_{1}\|w\|_{2}^{2}+2 \mu_{0}\left(A_{p} u_{\infty}^{1}-A_{p} u_{\infty}^{2}, u_{\infty}^{1}-u_{\infty}^{2}\right)+b\left(w, u_{\infty}^{2}, w\right)=<F\left(u_{\infty}^{1}\right)-F\left(u_{\infty}^{2}\right), w>.
$$

Here we used the relation

$$
\left(B\left(u_{\infty}^{1}, u_{\infty}^{1}\right)-B\left(u_{\infty}^{2}, u_{\infty}^{2}\right), u_{\infty}^{1}-u_{\infty}^{2}\right)=b\left(u_{\infty}^{1}-u_{\infty}^{2}, u_{\infty}^{2}, u_{\infty}^{1}-u_{\infty}^{2}\right)
$$

which can be proved by using the properties stated in (4). By using (7) we deduce from (12) that

$$
\mu_{1}|| w\left\|_{2}^{2} \leq\left|b\left(w, u_{\infty}^{2}, w\right)\right|+\beta\right\| w \|_{2}^{2} .
$$

thanks to (5) and (2), we have

$$
\mu_{1}\|w\|_{2}^{2} \leq C_{0} \lambda^{-\frac{1}{2}}\|w\|_{2}^{2}\left\|u_{\infty}^{2}\right\|_{2}+\beta\|w\|_{2}^{2}
$$

Invoking (10), we derive from this last estimate that

$$
\mu_{1}\|w\|_{2}^{2} \leq C_{0} \lambda^{-\frac{1}{2}}\|w\|_{2}^{2} \frac{\|F(0)\|_{V^{*}}}{\mu_{1}-\beta}+\beta\|w\|_{2}^{2},
$$

or equivalently

$$
\left(\mu_{1}-\frac{C_{0}\|F(0)\|_{V^{*}}}{\sqrt{\lambda_{1}}\left(\mu_{1}-\beta\right)}-\beta\right)\|w\|_{2}^{2} \leq 0 .
$$

Thanks to (11), this last estimate implies that $w=0$.

Let us introduce some assumptions on the mapping $G$. We suppose that:

Conditions $G 1$. for any $t \in[0, T]$ and $v \in H$,

$$
\|G(t, v)\|_{L_{2}^{0,0}}^{2} \leq \gamma(t)(\zeta+\rho(t))\left\|v-u_{\infty}\right\|
$$

where $\zeta$ is a positive constant and $\gamma(t), \rho(t)$ are nonnegative integrable functions such that there exist real numbers $\theta>0, M_{\gamma} \geq 1, M_{\rho} \geq 1$ with

$$
\gamma(t) \leq M_{\gamma} e^{-\theta t}, \rho(t) \leq M_{\rho} e^{-\theta t}, t \geq 0 .
$$

The first main result of the paper is given below.

Theorem 3.2. Let $u_{\infty} \in V$ be a stationary solution to (9). Suppose that Conditions F1 and G1 hold. We assume also that

$$
2 \mu_{1}>\lambda^{-2} \zeta+\frac{2 C_{0}}{\sqrt{\lambda_{1}}}\left\|u_{\infty}\right\|_{2}+2 \beta
$$

Then any weak solution $u(t)$ of (3) converges to the solution $u_{\infty}$ of (9) exponentially in mean square. Equivalently, there exist real numbers $a>0$ and $M_{0}=M_{0}\left(u_{0}\right)>0$ such that

$$
\mathbb{E}|| u(t)-u_{\infty} \|^{2} \leq M_{0} e^{-a t}, t \geq 0 .
$$

Proof. Since $2 \mu_{1}>\lambda^{-2} \zeta+\frac{2 C_{0}}{\sqrt{\lambda_{1}}}\left\|u_{\infty}\right\|_{2}+2 \beta$, we can choose a real number $a \in(0, \theta)$ such that $2 \mu_{1}>\lambda^{-2}(\zeta+a)+\frac{2 C_{0}}{\sqrt{\lambda_{1}}}\left\|u_{\infty}\right\|_{2}+2 \beta$. By Itô's formula we have

$$
\begin{array}{r}
e^{a t}\left\|u(t)-u_{\infty}\right\|^{2}=\left\|u_{0}-u_{\infty}\right\|^{2}+a \int_{0}^{t} e^{a s}\left\|u(s)-u_{\infty}\right\|^{2} d s-2 \mu_{1} \int_{0}^{t} e^{a s}\left(A u(s), u(s)-u_{\infty}\right) d s \\
-4 \mu_{0} \int_{0}^{t} e^{a s}<A_{p} u(s), u(s)-u_{\infty}>d s+2 \int_{0}^{t} e^{a s}<F(u(s)), u(s)-u_{\infty}>d s \\
-2 \int_{0}^{t} e^{a s}<B(u(s), u(s)), u(s)-u_{\infty}>d s+\int_{0}^{t} e^{a s}\|G(s, u(s))\|_{L_{2}^{0,0}}^{2} d s \\
+2 \int_{0}^{t} e^{a s}\left(G(s, u(s)), u(s)-u_{\infty}\right) d W .
\end{array}
$$


Taking the mathematical expectation to both sides of this equations yields

$$
\begin{array}{r}
e^{a t} \mathbb{E}\left\|u(t)-u_{\infty}\right\|^{2}=\mathbb{E}\left\|u_{0}-u_{\infty}\right\|^{2}+\int_{0}^{t} e^{a s} \mathbb{E}\|G(s, u(s))\|_{L_{2}^{0,0}}^{2} d s+a \int_{0}^{t} e^{a s} \mathbb{E}\left\|u(s)-u_{\infty}\right\|^{2} d s \\
-\int_{0}^{t} 2 e^{a s} \mathbb{E}\left(\mu_{1}\left(A u(s), u(s)-u_{\infty}\right)+2 \mu_{0}<A_{p} u(s), u(s)-u_{\infty}>\right) d s \\
-2 \int_{0}^{t} e^{a s} \mathbb{E}<B(u(s), u(s)), u(s)-u_{\infty}>d s \\
+2 \int_{0}^{t} e^{a s} \mathbb{E}<F(u(s)), u(s)-u_{\infty}>d s .
\end{array}
$$

Since $u_{\infty}$ is a stationary solution to (9) then there holds

$$
\begin{array}{r}
2 \int_{0}^{t} e^{a s} \mathbb{E}<F(u(s)), u(s)-u_{\infty}>d s=2 \int_{0}^{t} e^{a s} \mathbb{E}<B(u(s), u(s)), u(s)-u_{\infty}>d s \\
+4 \mu_{0} \int_{0}^{t} e^{a s} \mathbb{E}<A_{p} u(s), u(s)-u_{\infty}>d s \\
+2 \mu_{1} \int_{0}^{t} e^{a s} \mathbb{E}\left(A u(s), u(s)-u_{\infty}\right) d s .
\end{array}
$$

By using the last equation and the identity (13), we obtain from (16) that

$$
\begin{array}{r}
e^{a t} \mathbb{E}|| u(t)-u_{\infty} \|^{2}= \\
-2 \int_{0}^{t} e^{a s} \mathbb{E}\left(2 \mu_{0}<u_{p} u(s)-u_{\infty}\left\|_{\infty}, u(s)-u_{\infty}^{t} e^{a s} \mathbb{E}|| u(s)-u_{\infty}\right\|^{2} d s+\int_{0}^{t} e^{a s} \mathbb{E}\|G(s, u(s))\|_{L_{2}^{0,0}}^{2} d s\right. \\
\left.+2 \int_{0}^{t} e^{a s} \mathbb{E}<F(s)-u_{\infty} \|_{2}^{2}\right) d s \\
-2 \int_{0}^{t} e^{a s} \mathbb{E} b\left(u(s)-u_{\infty}, u_{\infty}, u(s)-u_{\infty}\right) d s,
\end{array}
$$

from which along with (7), (5) and Conditions F1 we obtain that

$$
\begin{array}{r}
e^{a t} \mathbb{E}\left\|u(t)-u_{\infty}\right\|^{2} \leq \mathbb{E}\left\|u_{0}-u_{\infty}\right\|^{2}+a \int_{0}^{t} e^{a s} \mathbb{E}|| u(s)-u_{\infty}\left\|^{2} d s-2 \mu_{1} \int_{0}^{t} e^{a s} \mathbb{E}|| u(s)-u_{\infty}\right\|_{2}^{2} d s \\
+2 \beta \int_{0}^{t} e^{a s} \mathbb{E}\left\|u(s)-u_{\infty}\right\|_{2}^{2} d s+\int_{0}^{t} e^{a s} \mathbb{E}\|G(s, u(s))\|_{L_{2}^{0,0}}^{2} d s \\
+\frac{2 C_{0}\left\|u_{\infty}\right\|_{2}}{\sqrt{\lambda_{1}}} \int_{0}^{t} e^{a s} \mathbb{E}\left\|u(s)-u_{\infty}\right\|_{2}^{2} d s .
\end{array}
$$

Using Conditions G1 and (2), we infer from (17) that

$$
\begin{array}{r}
e^{a t} \mathbb{E}\left\|u(t)-u_{\infty}\right\|^{2} \leq\left(\lambda^{-2}(a\right. \\
\left.+\zeta)+2 \beta+\frac{2 C_{0}\left\|u_{\infty}\right\|_{2}}{\sqrt{\lambda_{1}}}-2 \mu_{1}\right) \int_{0}^{t} e^{a s} \mathbb{E}\left\|u(s)-u_{\infty}\right\|_{2}^{2} d s \\
+\mathbb{E}\left\|u_{0}-u_{\infty}\right\|^{2}+\int_{0}^{t} e^{a s}\left(\gamma(s)+\rho(s) \mathbb{E}\left\|u(s)-u_{\infty}\right\|^{2}\right) d s .
\end{array}
$$

Since $\lambda^{-2}(a+\zeta)+2 \beta+\frac{2 C_{0}\left\|u_{\infty}\right\|_{2}}{\sqrt{\lambda_{1}}}-2 \mu_{1}<0$, we have that

$$
e^{a t} \mathbb{E}|| u(t)-u_{\infty}\left\|^{2} \leq \mathbb{E}|| u_{0}-u_{\infty}\right\|^{2}+\int_{0}^{t} e^{a s}\left(\gamma(s)+\rho(s) \mathbb{E}\left\|u(s)-u_{\infty}\right\|^{2}\right) d s .
$$


As $\theta>0$, we can apply Gronwall's inequality and conclude by using assumptions on $\gamma(t)$ and $\rho(t)$ that there exists a constant $M_{0}=M_{0}\left(u_{0}\right)$ such that

$$
\mathbb{E}|| u(t)-u_{\infty} \|^{2} \leq M_{0} e^{-a t}, t \geq 0 .
$$

And this ends the proof of the theorem.

Referring to exponential convergence with probability one of the weak solution we have the following result.

Theorem 3.3. Any weak solution $u(t)$ of (3) converges to the stationary solution $u_{\infty}$ of (9) almost surely exponentially provided that all the assumptions of Theorem 3.1 are satisfied.

Proof. Let $N$ be a nonnegative number and $t \geq N$. By Itô's formula we have

$$
\begin{array}{r}
\left\|u(t)-u_{\infty}\right\|^{2}=\left\|u(N)-u_{\infty}\right\|^{2}-4 \mu_{0} \int_{N}^{t}<A_{p} u(s)-A_{p} u_{\infty}, u(s)-u_{\infty}>d s \\
-2 \mu_{1} \int_{N}^{t}\left\|u(s)-u_{\infty}\right\|_{2}^{2} d s-2 \int_{N}^{t} b\left(u(s)-u_{\infty}, u_{\infty}, u(s)-u_{\infty}\right) d s \\
+\int_{N}^{t}\|G(s, u(s))\|_{L_{2}^{0,0}}^{2} d s+\int_{N}^{t}\left(G(s, u(s)), u(s)-u_{\infty}\right) d W \\
+2 \int_{N}^{t}<F(u(s))-F\left(u_{\infty}\right), u(s)-u_{\infty}>d s .
\end{array}
$$

Let us set $\Lambda_{N}(t)=2 \int_{N}^{t}\left(G\left(s, u(s), u(s)-u_{\infty}\right) d W\right.$. By Burkhölder-Davis-Gundy's inequality we have

$$
\begin{aligned}
\mathbb{E} \sup _{N \leq t \leq N+1} \Lambda_{N}(t) & \leq \eta_{1} \mathbb{E}\left(\int_{N}^{N+1}\left\|u(s)-u_{\infty}\right\|^{2}\|G(s, u(s))\|_{L_{2}^{0,0}}^{2} d s\right)^{\frac{1}{2}}, \\
& \leq \eta_{1} \mathbb{E}\left(\sup _{N \leq t \leq N+1}\left\|u(t)-u_{\infty}\right\|^{2} \int_{N}^{N+1}\|G(s, u(s))\|_{L_{2}^{0,0}}^{2} d s\right)^{\frac{1}{2}}, \\
& \leq \eta_{2} \int_{N}^{N+1} \mathbb{E}\|G(s, u(s))\|_{L_{2}^{0,0}}^{2} d s+\frac{1}{2} \mathbb{E} \sup _{N \leq t \leq N+1}\left\|u(t)-u_{\infty}\right\|^{2},
\end{aligned}
$$

where $\eta_{1}, \eta_{2}>0$. From this we deduce that there exists $\eta_{0}$ such that

$$
\begin{aligned}
\mathbb{E} \sup _{N \leq t \leq N+1}\left\|u(t)-u_{\infty}\right\|^{2} \leq \mathbb{E}\left\|u(N)-u_{\infty}\right\|^{2}+2 \frac{C_{0}\left\|u_{\infty}\right\|_{2}}{\sqrt{\lambda_{1}}} \int_{N}^{N+1} \mathbb{E}\left\|u(s)-u_{\infty}\right\|_{2}^{2} d s \\
-2 \mu_{1} \int_{N}^{N+1} \mathbb{E}\left\|u(s)-u_{\infty}\right\|_{2}^{2} d s+\beta \int_{N}^{N+1} \mathbb{E}\left\|u(s)-u_{\infty}\right\|_{2}^{2} d s \\
+\eta_{0} \int_{N}^{N+1} \mathbb{E}\|G(s, u(s))\|_{L_{2}^{0,0}}^{2} d s+\frac{1}{2} \mathbb{E} \sup _{N \leq t \leq N+1}\left\|u(t)-u_{\infty}\right\|^{2},
\end{aligned}
$$

Using the assumptions of the theorem, we readily check from this that

$$
\frac{1}{2} \mathbb{E} \sup _{N \leq t \leq N+1}\left\|u(t)-u_{\infty}\right\|^{2} \leq \mathbb{E}\left\|u(N)-u_{\infty}\right\|^{2}+\eta_{0} \int_{N}^{N+1}\left(\gamma(s)+(\zeta+\rho(s)) \mathbb{E}\left\|u(s)-u_{\infty}\right\|^{2}\right) d s .
$$

Due to the assumptions on $\gamma(),. \rho($.$) and the fact that a \in(0, \theta), M_{\gamma} \geq 1, M_{\rho} \geq 1$ we obtain as in Theorem 3.1 that there exists $M_{1}=M_{1}\left(u_{0}\right)$ such that

$$
\mathbb{E} \sup _{N \leq t \leq N+1}\left\|u(t)-u_{\infty}\right\|^{2} \leq M_{1} e^{-a N} .
$$

Now, the proof of the theorem follows from the Borel-Cantelli lemma. 
Theorem 3.4. Let $u_{\infty}$ be the unique stationary solution to (9). Assume that Conditions F1 and the following hold:

$$
\begin{aligned}
& G\left(t, u_{\infty}\right)=0, t \geq 0, \\
& \|G(t, u)-G(t, v)\|_{L_{2}^{0}} \leq C_{G}\|u-v\|_{2}, C_{G}>0, \forall u, v \in V .
\end{aligned}
$$

If $2 \mu_{1}>\frac{2 C_{0}\left\|u_{\infty}\right\|_{2}}{\sqrt{\lambda_{1}}}+2 \beta+C_{G}^{2}$, then any weak solution $u(t)$ to (3) converges to $u_{\infty}$ exponentially in mean square. Moreover, $u_{\infty}$ is exponentially stable in mean square, that is, there exists a real number $\eta>0$ such that

$$
\mathbb{E}|| u(t)-u_{\infty}\left\|^{2} \leq \mathbb{E}\right\| u_{0}-u_{\infty} \|^{2} e^{-\eta}, t \geq 0 .
$$

Pathwise exponential stability with probability one also holds.

Proof. We can check that

$$
\begin{array}{r}
u(t)-u_{\infty}=u_{0}-u_{\infty}-\mu_{1} \int_{0}^{t} A\left(u(s)-u_{\infty}\right) d s-2 \mu_{0} \int_{0}^{t}\left(A_{p} u(s)-A_{p} u_{\infty}\right) d s \\
-\int_{0}^{t}\left[B(u(s), u(s))-B\left(u_{\infty}, u_{\infty}\right)\right] d s+\int_{0}^{t}\left[F(u(s))-F\left(u_{\infty}\right)\right] d s \\
+\int_{0}^{t}\left[G(s, u(s))-G\left(s, u_{\infty}\right)\right] d W .
\end{array}
$$

Let $\eta>0$ be a real number to be fixed later. By Itô's formula, we have

$$
\begin{array}{r}
e^{\eta t}|| u(t)-u_{\infty}\left\|^{2}=\right\| u_{0}-u_{\infty}\left\|^{2}+\eta \int_{0}^{t} e^{\eta s}|| u(s)-u_{\infty}\right\|^{2} d s-2 \mu_{1} \int_{0}^{t} e^{\eta s}|| u(s)-u_{\infty} \|_{2}^{2} d s \\
-4 \mu_{0} \int_{0}^{t} e^{\eta s}\left(A_{p} u(s)-A_{p} u_{\infty}, u(s)-u_{\infty}\right) d s+\int_{0}^{t} e^{\eta s}|| G\left(s, u(s)-G\left(s, u_{\infty}\right) \|_{L_{2}^{0,0}}^{2} d s\right. \\
-2 \int_{0}^{t} e^{\eta s}\left(B(u(s), u(s))-B\left(u_{\infty}, u_{\infty}\right), u(s)-u_{\infty}\right) d s \\
+2 \int_{0}^{t} e^{\eta s}\left(G(s, u(s))-G\left(s, u_{\infty}\right), u(s)-u_{\infty}\right) d W \\
+2 \int_{0}^{t} e^{\eta s}\left(F(u(s))-F\left(u_{\infty}\right), u(s)-u_{\infty}\right) d s .
\end{array}
$$

Taking the mathematical expectation to both sides yields

$$
\begin{array}{r}
e^{\eta t} \mathbb{E}\left\|u(t)-u_{\infty}\right\|^{2}=\mathbb{E}\left\|u_{0}-u_{\infty}\right\|^{2}+\eta \int_{0}^{t} e^{\eta s} \mathbb{E}|| u(s)-u_{\infty}\left\|^{2} d s-2 \mu_{1} \int_{0}^{t} e^{\eta s} \mathbb{E}\right\| u(s)-u_{\infty} \|_{2}^{2} d s \\
+2 \int_{0}^{t} e^{\eta s} \mathbb{E}\left[\left(F(u(s))-F\left(u_{\infty}\right), u(s)-u_{\infty}\right)+\| G\left(s, u(s)-G\left(s, u_{\infty}\right) \|_{L_{2}^{0,0}}^{2}\right] d s\right. \\
-2 \int_{0}^{t} e^{\eta s} \mathbb{E}\left(B(u(s), u(s))-B\left(u_{\infty}, u_{\infty}\right), u(s)-u_{\infty}\right) d s \\
-4 \mu_{0} \int_{0}^{t} e^{\eta s} \mathbb{E}\left(A_{p} u(s)-A_{p} u_{\infty}, u(s)-u_{\infty}\right) d s .
\end{array}
$$

By (5), (7) and the assumptions on $F$ and $G$, we have that

$$
\begin{array}{r}
e^{\eta t} \mathbb{E}\left\|u(t)-u_{\infty}\right\|^{2} \leq\left(\eta \frac{1}{\lambda^{2}}+\frac{2 C_{0}\left\|u_{\infty}\right\|_{2}}{\sqrt{\lambda_{1}}}+2 \beta+C_{G}^{2}-2 \mu_{1}\right) \int_{0}^{t} e^{\eta s} \mathbb{E}\left\|u(s)-u_{\infty}\right\|_{2}^{2} d s \\
+\mathbb{E}\left\|u_{0}-u_{\infty}\right\|^{2} .
\end{array}
$$

As $2 \mu_{1}>\frac{2 C_{0}\left\|u_{\infty}\right\|_{2}}{\sqrt{\lambda_{1}}}+2 \beta+C_{G}^{2}$, we choose $\eta$ such that $\eta \lambda^{-2}+\frac{2 C_{0}\left\|u_{\infty}\right\|_{2}}{\sqrt{\lambda_{1}}}+2 \beta+C_{G}^{2}-2 \mu_{1}<0$. This choice and (18) complete the proof of the exponential stability in mean square. The proof 
of the exponential stability with probability one follows from a similar method to the one in Theorem 3.3.

Theorem 3.5. We assume that there exists $\zeta>0$ such that

$$
\|G(t, u)\|_{L_{2}^{0,0}}^{2} \leq \gamma(t)+(\zeta+\rho(t))\|u\|^{2},
$$

where $\gamma(t)$ and $\rho(t)$ satisfy the conditions given in Theorem 3.1.

We also suppose that $F:[0, \infty] \times V \rightarrow V^{*}$ verifies

$$
|<F(t, v), v>| \leq \alpha(t)+\left(C_{F}+\beta(t)\right)\|v\|^{2},
$$

where $C_{F}>0$, and $\alpha(t), \beta(t)$ are integrable functions such that there exist real numbers $\delta>0$, $M_{\alpha} \geq 1, M_{\beta} \geq 1$ with $\alpha(t) \leq M_{\alpha} e^{-\delta t}, \beta(t) \leq M_{\beta} e^{-\delta t}, t \geq 0$. If in addition we assume that $2 \lambda_{1}\left(\mu_{1} \lambda_{1}+2 \mu_{0} K_{0}\right)>\zeta+2 C_{F}$, then any weak solution $u(t)$ to (3) converges to zero exponentially with probability one.

Proof. Let $\eta \in(0, \delta)$ be such that $-2 \lambda_{1}\left(\mu_{1} \lambda_{1}+2 \mu_{0} K_{0}\right)+\eta+2 C_{F}+\zeta<0$. By Itô's formula we have

$$
\begin{array}{r}
e^{\eta t} \mathbb{E}\|u(t)\|^{2}=\mathbb{E}\left\|u_{0}\right\|^{2}+\int_{0}^{t} e^{\eta s} \mathbb{E}\left[\eta\|u(s)\|^{2}-2 \mu_{1}\|u(s)\|_{2}^{2}+\| G\left(s, u(s) \|_{L_{2}^{0,0}}^{2}\right] d s\right. \\
-2 \int_{0}^{t} e^{\eta s} \mathbb{E}\left[2 \mu_{0}<A_{p} u(s), u(s)>-<B(u(s), u(s)), u(s)>\right] d s \\
+2 \int_{0}^{t} e^{\eta s} \mathbb{E}<F(s, u(s)), u(s)>d s .
\end{array}
$$

By using (7), (2), and the assumptions on $F$ and $G$ we obtain that

$$
\begin{aligned}
e^{\eta t} \mathbb{E}\|u(t)\|^{2} \leq \mathbb{E}\left\|u_{0}\right\|^{2}+(\eta & \left.+2 C_{F}+\zeta-2 \lambda_{1}\left(\mu_{1} \lambda_{1}+4 \mu_{0} K_{0}\right)\right) \int_{0}^{t} e^{\eta s} \mathbb{E}\|u(s)\|^{2} d s \\
& +\int_{0}^{t}\left(\gamma(s)+2 \alpha(s)+[2 \beta(s)+\rho(s)] \mathbb{E}\|u(s)\|^{2}\right) e^{\eta s} d s
\end{aligned}
$$

Since $-2 \lambda_{1}\left(\mu_{1} \lambda_{1}+4 \mu_{0} K_{0}\right)+\eta+2 C_{F}+\zeta<0$, we have that

$$
e^{\eta t} \mathbb{E}|| u(t)\left\|^{2} \leq \mathbb{E}|| u_{0}\right\|^{2}+\int_{0}^{t}\left(\gamma(s)+2 \alpha(s)+[2 \beta(s)+\rho(s)] \mathbb{E}\|u(s)\|^{2}\right) e^{\eta s} d s .
$$

We deduce from this and Gronwall's lemma that any weak solution to (3) converges to zero exponentially in mean square. We finish the proof by the same method as in the proof of Theorem (3.3).

\section{Stabilization}

In this section we briefly discuss the stabilization of the stochastic evolution equations (3). According to the analysis in [12] (see also [14]) it is enough to consider a one dimensional Wiener process for that purpose. Throughout we suppose that $G(t, v)=\sigma\left(v-u_{\infty}\right), v \in H$, and that

Conditions F2:

$$
\begin{aligned}
& F(0) \neq 0, \\
& \|F(u)-F(v)\| \leq C_{F}\|u-v\|, \forall u, v \in H .
\end{aligned}
$$

Lemma 4.1. Let $u_{\infty}$ be the unique stationary solution to (9). If Conditions F2 hold and

$$
\lambda_{1}\left(2 \mu_{0} K_{0}+\mu_{1} \lambda_{1}\right)>C_{0} \lambda^{\frac{3}{2}}\left\|u_{\infty}\right\|_{2}+C_{F},
$$

then the stationary solution $u_{\infty}$ is stable. 
Proof. The proof is similar to the one in [22] where the authors imposed a condition (see equation (29) in page 67 of [22]) that, in our belief, is much more stronger that (19). So for the convenience of the reader we give the detail of the proof of the above lemma. Let $v(t)$ be the weak solution of the deterministic evolution equations

$$
\frac{\partial v(t)}{\partial t}+\mu_{1} A v(t)+2 \mu-0 A_{p} v(t)+B(v(t), v(t))=F(v(t)) .
$$

Let $w(t)=v(t)-u_{\infty}$. It is easily seen that $w(t)$ satisfies

$$
\begin{array}{r}
\frac{d}{d t}\|w(t)\|^{2}+2 \mu_{1}\|w(t)\|_{2}^{2}+4 \mu_{0}<A_{p} v(t)-A_{p} u_{\infty}, w(t)>+2 b\left(w(t), u_{\infty}, w(t)\right) \\
=2\left(F(v(t))-F\left(u_{\infty}\right), w(t)\right)
\end{array}
$$

Employing (7) and (5) along with (2) in the above equation implies that

$$
\frac{d}{d t}\|w(t)\|^{2}+\left(2 \mu_{1} \lambda_{1}^{2}-2 C_{0} \lambda_{1}^{\frac{3}{2}}\left\|u_{\infty}\right\|_{2}-2 C_{F}+4 \mu_{0} K_{0} \lambda_{1}\right)\left|\||| w(t)\|^{2} \|^{2} \leq 0 .\right.
$$

As $R=2 \mu_{1} \lambda_{1}^{2}-2 C_{0} \lambda_{1}^{\frac{3}{2}}\left\|u_{\infty}\right\|_{2}-2 C_{F}+4 \mu_{0} K_{0} \lambda_{1}>0$, we obtain by using Gronwall's lemma that

$$
\frac{d}{d t}\|w(t)\|^{2} \leq\|w(0)\|^{2} e^{-R t}, \forall t \geq 0
$$

This proves the lemma.

It follows from the above lemma that if $\lambda_{1}\left(2 \mu_{0} K_{0}+\mu_{1} \lambda_{1}\right) \leq C_{0} \lambda_{1}^{\frac{3}{2}}\left\|u_{\infty}\right\|_{2}+C_{F}$, then we do not know whether the stationary solution $u_{\infty}$ is stable or not. However, we have the following stabilization result.

Theorem 4.2. Let $u_{\infty}$ be the unique stationary solution of (9) and $u(t)$ is a weak solution to (3). Let $C_{1}:=\lambda_{1}^{2} \mu_{1}-C_{0} \lambda_{1}^{\frac{3}{2}}\left\|u_{\infty}\right\|_{2}>0, C_{2}:=\lambda_{1}^{2} \mu_{1}+2 \mu_{0} K_{0} \lambda_{1}-2 C_{0} \lambda_{1}^{\frac{3}{2}}\left\|u_{\infty}\right\|_{2}$ and

$$
\lambda_{1}\left(\mu_{1} \lambda_{1}+2 \mu_{0} K_{0}\right) \leq C_{0} \lambda_{1}^{\frac{3}{2}}\left\|u_{\infty}\right\|_{2}+C_{F} .
$$

Assume that $\sigma$ is a real number such that $2 C_{2}+\sigma^{2}>2 C_{F}$. If Conditions F2 holds, then there exists $\Omega_{0} \subset \Omega$ with $\mathbb{P}\left(\Omega_{0}\right)=1$ and for any $\omega \in \Omega \backslash \Omega_{0}$ there exists $T(\omega)>0$ such that

$$
\left\|u(t)-u_{\infty}\right\|^{2} \leq\left\|u_{0}-u_{\infty}\right\|^{2} e^{-\eta t}, \forall t \geq T(\omega) .
$$

Here $\eta=\frac{1}{2}\left(\sigma^{2}+2 C_{2}-2 C_{F}\right)>0$ and the function $G$ is given by $G(t, v)=\sigma\left(v-u_{\infty}\right)$ for any $v \in H$.

Proof. By Itô's formula we have that

$$
\begin{array}{r}
\left\|u(t)-u_{\infty}\right\|^{2}=\left\|u_{0}-u_{\infty}\right\|^{2}-4 \mu_{0} \int_{0}^{t}<A_{p} u(s), u(s)-u_{\infty}>d s+\int_{0}^{t}\|G(s, u(s))\|_{L_{2}^{0,0}}^{2} d s \\
-2 \mu_{1} \int_{0}^{t}\left(A u(s), u(s)-u_{\infty}\right) d s-2 \int_{0}^{t}<B(u(s), u(s)), u(s)-u_{\infty}>d s \\
+2 \int_{0}^{t}<F(u(s)), u(s)-u_{\infty}>d s+2 \int_{0}^{t}\left(G(s, u(s)), u(s)-u_{\infty}\right) d W
\end{array}
$$


Arguing as in the proof of Theorem 3.1, we can show that

$$
\begin{array}{r}
\left\|u(t)-u_{\infty}\right\|^{2}=\left\|u_{0}-u_{\infty}\right\|^{2}-2 \mu_{1} \int_{0}^{t}\left\|u(s)-u_{\infty}\right\|_{2}^{2} d s+\int_{0}^{t}\|G(s, u(s))\|_{L_{2}^{0,0}}^{2} d s \\
+2 \int_{0}^{t}<F(u(s))-F\left(u_{\infty}\right), u(s)-u_{\infty}>d s \\
-4 \mu_{0} \int_{0}^{t}<A_{p} u(s)-A_{p} u_{\infty}, u(s)-u_{\infty}>d s \\
-2 \int_{0}^{t} b\left(u(s)-u_{\infty}, u_{\infty}, u(s)-u_{\infty}\right) d s \\
+2 \int_{0}^{t}\left(G(s, u(s)), u(s)-u_{\infty}\right) d W .
\end{array}
$$

Thanks to (7), (5) and the assumptions on $C_{1}$ we have that

$$
\begin{aligned}
& -4 \mu_{0} \int_{0}^{t}<A_{p} u(s)-A_{p} u_{\infty}, u(s)-u_{\infty}>d s+2 \int_{0}^{t}\left|b\left(u(s)-u_{\infty}, u_{\infty}, u(s)-u_{\infty}\right)\right| d s \\
\leq & 2 \mu_{1} \int_{0}^{t}\left\|u(s)-u_{\infty}\right\|_{2}^{2} d s+\left(-2 \mu_{1} \lambda_{1}^{2}-4 \mu_{0} K_{0} \lambda_{1}+\frac{2 C_{0}\left\|u_{\infty}\right\|_{2}}{\sqrt{\lambda_{1}}}\right) \int_{0}^{t}\left\|u(s)-u_{\infty}\right\|^{2} d s .
\end{aligned}
$$

By Itô's formula again, we get that

$$
\begin{array}{r}
\log \left\|u(t)-u_{\infty}\right\|^{2}=\log \left\|u_{0}-u_{\infty}\right\|^{2}+\int_{0}^{t} \frac{1}{\left\|u(s)-u_{\infty}\right\|^{2}}\left[-2 \mu_{1}\left\|u(s)-u_{\infty}\right\|_{2}^{2}\right. \\
-4 \mu_{0}<A_{p} u(s)-A_{p} u_{\infty}, u(s)-u_{\infty}>-2 b\left(u(s)-u_{\infty}, u_{\infty}, u(s)-u_{\infty}\right) \\
\left.+\sigma^{2}\left\|u(s)-u_{\infty}\right\|^{2}+2\left(F(u(s))-F\left(u_{\infty}\right), u(s)-u_{\infty}\right)\right] d s \\
+2 \int_{0}^{t} \frac{\sigma\left\|u(s)-u_{\infty}\right\|^{2}}{\left\|u(s)-u_{\infty}\right\|^{2}} d W-\frac{1}{2} \int_{0}^{t} \frac{4 \sigma^{2}\left\|u(s)-u_{\infty}\right\|^{4}}{\left\|u(s)-u_{\infty}\right\|^{4}} d s
\end{array}
$$

which along with (20) yield

$$
\log \left\|u(t)-u_{\infty}\right\|^{2} \leq \log \left\|u_{0}-u_{\infty}\right\|^{2}+\left(2 C_{F}-2 C_{2}-\sigma^{2}\right) t+2 \sigma W(t),
$$

where $C_{2}$ is given in the statement of the theorem.

As $\lim _{t \rightarrow \infty} \frac{W(t)}{t}=0$ almost surely, then there exists $\Omega_{0} \subset \Omega$ with $\mathbb{P}\left(\Omega_{0}\right)=1$ and for any $\omega \in \Omega \backslash \Omega_{0}$ there exists $T(\omega)>0$ such that for any $t \geq T(\omega)$

$$
\frac{2 \sigma W(t)}{t} \leq \frac{1}{2}\left(\sigma^{2}+2 C_{2}-2 C_{F}\right)
$$

Thus for any $\omega \in \Omega \backslash \Omega_{0}$ there exists $T(\omega)>0$ such that for any $t \geq T(\omega)$

$$
\log \left\|u(t)-u_{\infty}\right\|^{2} \leq \log \left\|u_{0}-u_{\infty}\right\|^{2}+\frac{1}{2}\left(2 C_{F}-2 C_{2}-\sigma^{2}\right) t
$$

Equivalently, there exists $\Omega_{0} \subset \Omega$ with $\mathbb{P}\left(\Omega_{0}\right)=1$ and for any $\omega \in \Omega \backslash \Omega_{0}$ there exists $T(\omega)>0$ such that

$$
\left\|u(t)-u_{\infty}\right\|^{2} \leq\left\|u_{0}-u_{\infty}\right\|^{2} e^{-\eta t}, t \geq T(\omega),
$$

where $\eta=\frac{1}{2}\left(\sigma^{2}+2 C_{2}-C_{F}\right)>0$. This completes the proof.

\section{ACKNOWLEDGMENT}

The research of the authors is supported by the University of Pretoria and the National Research Foundation South Africa. The first author is also grateful to the Abdu Salam ICTP, Trieste, Italy for its generous funding. 


\section{REFERENCES}

[1] H. Bellout, F. Bloom and J. Necas. Phenomenological behavior of multipolar viscous fluids. Quarterly of Applied Mathematics 50:559-583, 1992.

[2] H. Bellout, F. Bloom and J. Necas. Solutions for incompressible Non-Newtonian fluids. C. R. Acad. Sci. Paris Sér I. Math. 317:795-800,1993.

[3] H. Bellout, F. Bloom and J. Necas. Young measure-valued solutions for Non-Newtonian incompressible fluids. Communication in Partial Differential Equations. 19(11\& 12):1763-1803, 1994.

[4] H. Bellout, F. Bloom and J. Necas. Bounds for the dimensions of the attractors of nonlinear bipolar viscous fluids. Asymptotic Analysis. 11(2):131-167,1995.

[5] H. Bellout, F. Bloom and J. Necas. Existence, uniqueness and stability of solutions to initial boundary value problems for bipolar fluids. Differential and Integral Equations 8:453-464, 1995

[6] S. Albeverio, Z. Brzezniak and J.-L. Wu. Existence of global solutions and invariant measures for stochastic differential equations driven by Poisson type noise with non-Lipschitz coefficients.J. Math. Anal. Appl. 371(1):309-322, 2010.

[7] A. Bensoussan. Stochastic Navier-Stokes Equations. Acta Applicandae Mathematicae, 38:267-304, 1995.

[8] A. Bensoussan and R. Temam. Equations Stochastiques du Type Navier-Stokes. Journal of Functional Analysis, 13:195-222, 1973.

[9] H. Breckner Galerkin approximation and the strong solution of the Navier-Stokes equation. Journal of Applied Mathematics and Stochastic Analysis. 13(3):239-259, 2000.

[10] Z. Brzezniak and L. Debbi. On stochastic Burgers equation driven by a fractional Laplacian and space-time white noise. Stochastic differential equations: Theory and applications, Interdiscip. Math. Sci., 2, World Sci. Publ., pages 135-167, 2007.

[11] Z. Brzezniak, B. Maslowski and J. Seidler. Stochastic nonlinear beam equation, Probab. Theory Relat. Fields. 132(2):119-144, 2005.

[12] T. Caraballo, J.A. Langa and T. Taniguchi. The exponential behaviour and stabilizability of stochastic 2D-Navier-Stokes equations. J. Differential Equations. 179(2):714-737, 2002.

[13] T. Caraballo, J. Real and T. Taniguchi. On the exisrence and uniqueness of solutions to stochastic threedimensional Lagrangian averaged Navier-Stokes equations. Proc. R. Soc. Lond. Ser. A Math. Phys. Eng. Sci. 462(2066):459-479, 2006.

[14] T. Caraballo, A.M. Márquez-Dur'an and J. Real. The asymptotic behaviour of a stochastic 3D LANS- $\alpha$ model. Appl. Math. Optim. 53(2):141-161, 2006.

[15] J. Chen and Z.M. Chen. Stochastic Non-Newtonian fluid motion equations of anonlinear bipolar viscous fluid. Journal of Mathematical Analysis and Applications. 369:486-509, 2010.

[16] G. Da Prato and J. Zabczyk. Stochastic Equations in Infinite Dimensions. Cambridge University Press, 1992.

[17] G. Da Prato and A. Debussche 2D stochastic Navier-Stokes equations with a time-periodic forcing term. $J$. Dynam. Differential Equations 20(2):301-335, 2008.

[18] G. Deugoue and M. Sango. On the Stochastic 3D Navier-Stokes- $\alpha$ Model of Fluids Turbulence, Abstract and Applied Analysis, vol. 2009, Article ID 723236, 27 pages, 2009. doi:10.1155/2009/723236.

[19] G. Deugoue and M. Sango. On the Strong Solution for the 3D Stochastic Leray-Alpha Model, Boundary Value Problems, vol. 2010, Article ID 723018, 31 pages, 2010. doi:10.1155/2010/723018.

[20] Q. Du and M.D. Gunzburger. Analysis of Ladyzhenskaya Model for Incompressible Viscous Flow. Journal of Mathematical Analysis and Applications. 155:21-45, 1991.

[21] J. Freshe and M. Ruzicka. Non-homogeneous generalized Newtonian fluids. Mathematische Zeitschrift. 260(2):353-375, 2008.

[22] B. Guo and G. Lin. Existence and Uniqueness of stationary solutions of Non-Newtonian viscous incompressible fluids. Communications in nonlinear Science and Numerical Simulation. 4(1):63-68, 1999.

[23] B. Guo, C. Guo and J. Zhang. Martingale and stationary solutions for stochastic Non-Newtonian fluids. Differential and Integral Equations. 23(3 \& 4):303-326, 2010.

[24] O.A. Ladyzhensakya. The mathematical theory of viscous incompressible flow. Gordon and Breach, New York, 1969.

[25] O.A. Ladyzhensakya. New equations for the description of the viscous incompressible fluids and solvability in the large of the boundary value problems for them. In Boundary Value Problems of Mathematical Physics $V$. American Mathematical Society, Providence, RI, 1970.

[26] J.L. Lions. Quelques méthodes de résolution de probléms aux limites non-linéaires. Dunod, Paris, 1969.

[27] J. Malek, J. Necas and A. Novotny. Measure-valued solutions and asymptotic behavior of a multipolar model of a boundary layer. Czechoslovak Mathematical Journal. 42(3):549-576, 1992.

[28] J. Malek, J. Necas, M. Rokyta and M. Ruzicka. Weak and measure-valued solutions to evolutionary PDEs. Applied Mathematics and Mathematical Computation, 13. Chapman \& Hall, London, 1996.

[29] R. Mikulevicius and B.L. Rozovskii. Stochastic Navier-Stokes Equations and Turbulent Flows. SIAM J. Math. Anal., 35(5):1250-1310, 2004. 
[30] J. Necas and M. Silhavy Multipolar viscous fluids. Quaterly of Applied Mathematics. XLIX(2):247-266, 1991.

[31] J. Necas, A. Novotny and M. Silhavy. Global solution to the compressible isothermal multipolar fluids. $J$. Math. Anal. Appl. 162:223-242, 1991.

[32] E. Pardoux. Equations aux dérivées partielles stochastiques monotones. Thèse de Doctorat, Université ParisSud, 1975.

[33] S. Peszat and J. Zabczyk. Stochastic Partial Differential Equations with Levy Noise. An evolution equation approach. Encyclopedia of Mathematics and its Applications 113, Cambridge university Press, 2007.

[34] P. A. Razafimandimby and M. Sango. Weak Solutions of a Stochastic Model for Two-Dimensional Second Grade Fluids, Boundary Value Problems, vol. 2010, Article ID 636140, 47 pages, 2010. doi:10.1155/2010/636140.

[35] P. A. Razafimandimby. On Stochastic Models Describing the Motions of Randomly Forced Linear Viscoelastic Fluids, Journal of Inequalities and Applications, vol. 2010, Article ID 932053, 27 pages, 2010. doi:10.1155/2010/932053.

[36] P.A. Razafimandimby and M. Sango. Asymptotic behavior of solutions of stochastic evolution equations for second grade fluids, In press C. R. Acad. Sci. Paris, Ser. I (2010), doi:10.1016/j.crma.2010.05.001

[37] M. Sango. Weak solutions for a doubly degenerate quasilinear parabolic equation with random forcing. Discrete Contin. Dyn. Syst. Ser. B, 7(4) (2007) 885-905.

[38] M. Sango. Magnetohydrodynamic turbulent flows: Existence results. Physica D: Nonlinear Phenomena 239(12): 912-923, 2010.

[39] M. Sango. Density dependent stochastic Navier-Stokes equations with non Lipschitz random forcing. Reviews in Mathematical Physics 22(6):669-697, 2010.

[40] R. Temam. Navier-Stokes Equations. North-Holland, 1979.

(P. A. Razafimandimby and M. Sango) Department of Mathematics and Applied Mathematics, University of Pretoria, Lynwood Road, Pretoria 0002, South Africa

Current address, P. A. Razafimandimby: Mathematics Section, The Abdu Salam International Center for Theoretical Physics, Strada Costiera, 11, I - 34151 Trieste, Italy

E-mail address, P.A. RAZAFIMANDIMBY: prazafim@ictp.it,paulrazafi@gmail.com

E-mail address, M. SANGO: mamadou.sango@up.ac.za 